\title{
Pregabalin lindert neuropathische Schmerzen nach Rückenmarksläsionen
}

Fragestellung: Ist Pregabalin bei Patienten mit chronisch neuropathischen Schmerzen nach inkomplettem oder komplettem spinalem Querschnittssyndrom wirksam?

Hintergrund: Zwei Drittel aller Patienten mit inkomplettem oder komplettem spinalem Querschnitt entwickeln im Lauf der Zeit chronische neuropathische Schmerzen, wobei diese sowohl unterhalb wie oberhalb der Höhe der Querschnittsläsion wahrgenommen werden können. Ein Drittel der Patienten beschreibt die Schmerzen als schwerwiegend und sehr intensiv. Die Therapie erfolgt bisher meist unbefriedigend mit Antikonvulsiva wie Carbamazepin, trizyklischen Antidepressiva und mit Medikamenten zur Reduktion der Spastik. Pregabalin ist zur Behandlung von neuropathischen Schmerzen bei Polyneuropathien und zum Teil auch bei der postzosterischen Neuralgie zugelassen, nachdem eine Vielzahl von randomisierten placebokontrollierten Studien eine therapeutische Überlegenheit bei diesen neuropathischen Schmerzzuständen gezeigt hatte. Die vorliegende Studie sollte die Wirksamkeit von Pregabalin bei Patienten mit chronisch neuropathischen Schmerzen nach Rückenmarksläsionen belegen.

Patienten und Methodik: In die Studie wurden Patienten über 18 Jahre mit Querschnittslähmungen aufgenommen, bei denen die Läsion mehr als zwölf Monate zurücklag und die seit mehr als drei Monaten unter chronischen neuropathischen Schmerzen litten. Die Patienten mussten auf einer 11-Punkte-Schmerzskala mindestens einen Wert von vier haben.

Alle Patienten absolvierten eine vierwöchige Baselinephase, in der sie täglich Tagebuch führten. Dann erfolgten Randomisierung und Dosisoptimierung von Pregabalin über einen Zeitraum von vier Wochen. Die Initialdosis betrug $150 \mathrm{mg}$ Pregabalin pro Tag. Die Dosis konnte je nach Verträglichkeit bis auf $600 \mathrm{mg}$ am Tag erhöht werden. Anschließend erfolgte eine zwölfwöchige Behandlungsphase mit stabiler Dosis von Pregabalin. Anschließend wurde das Medikament über eine Woche ausgeschlichen. Der primäre Endpunkt war die mittlere Schmerzintensität über die zwölfwöchige Behandlungsphase. Sekundäre Endpunkte umfassten den mittleren Schmerz-Score über die gesamte Studiendauer, den Prozentsatz der Patienten mit einer mehr als $30 \%$ igen Reduktion der Schmerzintensität, eine globale Patienteneinschätzung und die Schlafqualität.

Cardenas DD, Nieshoff EC, Suda K et al. A randomized trial of pregabalin in patients with neuropathic pain due to spinal cord injury. Neurology 2013; 80: $533-9$ ten chronische Schmerzen. Bei der Mehrzahl der QuerschnittsSyndrome handelte es sich um die Folgen eines Unfalls. Für den primären Endpunkt war die Studie ebenso positiv wie für alle sekundären Endpunkte. Die Reduktion der mittleren Schmerzintensität lag in der Placebogruppe bei 1,07, in der Pregabalingruppe bei 1,66. Die Schmerzen waren im Vergleich zu Baseline unter Placebo um 1,22 Punkte reduziert, unter Pregabalin um 1,92. Die Zahl der Responder betrug 33 von 105 unter Placebo (31,4\%) und 48 von 105 (45,7\%) unter Pregabalin. Auch in der subjektiven Einschätzung der Patienten ergab sich eine Überlegenheit von Pregabalin, ebenso wie bei der Verbesserung der Schlafqualität. Die Nebenwirkungen waren typisch für den Einsatz von Pregabalin und umfassten Müdigkeit, Schwindel, periphere Ödeme, Mundtrockenheit und Gewichtszunahme.

Schlussfolgerungen: Pregabalin ist in einer durchschnittlichen Tagesdosis von $400 \mathrm{mg}$ bei der Behandlung von chronisch neuropathischen Schmerzen bei spinalem Querschnittssyndrom wirksam.

\section{- Kommentar von Hans-Christoph Diener, Essen}

\section{Weitere wichtige Therapieoption}

Diese große randomisierte Studie ist methodisch sehr gut durchgeführt. Sie benutzte zur Therapieevaluation ein sehr stringentes Maß, nämlich die Änderung der mittleren Schmerzintensität über die gesamte Behandlungsphase von zwölf Wochen hinweg. Bei vielen anderen Studien wird nur die letzte Woche der Behandlung mit der Baseline verglichen. Die durchschnittlich eingenommene Tagesdosis von $400 \mathrm{mg}$ Pregabalin zeigt, dass diese Dosis wahrscheinlich das beste Verhältnis für Wirkung und Nebenwirkung aufweist. Die Ergebnisse sind glaubwürdig, da auch alle sekundären Endpunkte eine Überlegenheit von Pregabalin zeigten. Für Studien zur Behandlung von chronisch neuropathischen Schmerzen werden Responder üblicherweise als Patienten mit einer Reduktion der Schmerzintensität um mindestens 30\% definiert. Dieses Ausmaß erreichten immerhin $45 \%$ der bis dahin weitgehend therapierefraktären Patienten. Die Numberneeded-to-treat für einen signifikanten Therapieerfolg betrug sieben. Die Nebenwirungen entsprachen den von Pregabalin bekannten unerwünschten Effekten. Die Zahl der Therapieabbrecher aufgrund von Nebenwirkungen war mit fünf Patienten unter Placebo und sechs Patienten unter Pregabalin vergleichbar hoch. Die hier vorliegende Studie belegt nun, dass mit Pregabalin eine weitere wichtige Therapieoption für Patienten mit chronisch neuropathischen Schmerzen nach inkompletten oder kompletten spinalem Querschnitt vorhanden ist. 